\title{
Aggregated Quantified Response Time Matrix Formulation (ARMF) —A New Quality of Service Paradigm Technique
}

\author{
Balika. J. Chelliah ${ }^{1 *}$, K. Vivekanandan ${ }^{2}$ \\ 1 Department of Computer Science and Engineering, SRM Univerisity, India. \\ ${ }^{2}$ Department of Computer Science and Engineering, Pondicherry Engineering College, Pondicherry, India. \\ * Corresponding author. Email: balika888@gmail.com
}

Manuscript submitted April 7, 2015; accepted July 18, 2015

doi: 10.17706/jsw.10.9.1070-1078

\begin{abstract}
Workflow management system, a technique that manages different types of processes/jobs gathered as a workflow, ensures that each task in the workflow is accomplished to proceed further. When the process happens between two parties, the client and the server, a binding agreement or contract is defined specifying the Quality of Service (QoS) metrics such as products/services to be delivered, the deadlines and the cost of services. The goal of QoS is to select the appropriate web services for the customers achieve their goals. The existing service applications pretend to be a self-contained unit of functionalities that requests and retrieve a qualified service using service oriented architecture. But they face difficulties in identifying the right quality of a service based on functionality. Hence the major factor in QoS specification is QoS modeling, which involves the task of identifying the factors suitable for a functional requirement. QoS-aware service composition significantly affects the quality of a composite service and hence enormous research has been performed in this area. However, existing methods are restricted to predefined workflows, which can sustain the limitations. The limitations include the lack of guarantee for the optimality on overall QoS, satisfaction of customers and the completeness of finding a composite service solution. The proposed approach uses an Aggregated Quantified Response time Matrix Formulation (ARMF) methodology. The ARMF predicts the unknown QoS values by implementing two algorithms, the Aggregated Quantified Matrix Formulation (AQMF) Technique and the Optimal Incremental Multi-Attributes property Filtering (OIMF). It provides the user-response matrix where every entry in the matrix depicts the QoS value of the service opted by the user. The experimental results show that the proposed approach largely outperforms the existing techniques in terms of quality and efficiency required for the customer services.
\end{abstract}

Key words: Quality of service, service oriented application, web services, user-response matrix, matrix formulation, QOS modeling.

\section{Introduction}

With the widespread usage of web service, analyzing the quality of each and every web service that suits the requirements of a client is time consuming. The improvement of facilitating techniques for Web services dynamically modifies the business processes on the Web. The Web services are software gears intended to sustain interoperable machine-to-machine interaction over the complex set of connections. They have engrossed wide courtesy from both industry and the academic world. This has lead to the creation of more versatile web services. The main advantage of web service is that they are loosely coupled and self 
describing software application that is widespread across the Internet with the help of standard protocol, the SOAP and WS techniques (WSDL and UDDI).

Amongst versatile web services with the same functionality, the best service is opted based on its quality. QoS (Quality of Service) for WSs is a set of non-functional properties that include features such as error probability, scalability, reliability, compliance, Disaster recovery, accessibility, backup, response time and the response probabilities, etc. Most of the service users are much apprehensive about the performance of WSs they use; QoS can be used for discerning between functionally comparable WSs. Service-oriented architecture provides base level architecture to achieve rapid service composition and functional processes. To achieve different system QoS requirements, it is possible to select an appropriate set of concrete services and compose them to achieve the QoS goals. The existing service applications pretend to be a self-contained unit of functionalities that requests and retrieve a qualified service using service oriented architecture. But they face difficulties in identifying the right quality of a service based on functionality. Hence the major factor in QoS specification is QoS modeling, which involves the task of identifying the factors suitable for a functional requirement.

A collaborative quality-of-service (QoS) prediction approach is introduced that implements the concept of neighborhood-integrated matrix factorization (NIMF) to select the best Web Service by using the benefits of the past usage experience of other service users. This way the authors, by Zheng, Ma, Lyu and King provide a suggestion to reduce the time and cost to evaluate the web services deployed by a third party. The collaborative prediction methodology also helps the service users to choose the best composition in spite of no/less knowledge about the service. The accuracy of this NIMF concept is verified in collaboration with Matrix Density and proved to be successful. But the fact is that the NIMF approach is restricted to client-side QoS value analysis.

\section{Real Time Scenario and Reviews}

Web Service, the important backbone of service oriented applications, are selected and consumed from a list of available services. Quality is the major concern for a variety of applications to achieve high end results and hence care must be taken to employ better selection criteria to choose the appropriate service. Evaluation must be performed accurately to analyze the non-functional properties such as error probability, the response time and the response probabilities are also considered to derive at the best solution for selecting a well qualified web service. Dario Bruneo, Salvatore Distefano, Francesco Longo and Marco Scarpa proposed a non-Markovian stochastic Petri net (NMSPN) [1] model for detailing the stochastic QoS evaluation to arrive at the cumulative distribution function (CDF) of the service response time and reliability of the composed web services.

Web services are highly brought into play due to its interoperable nature, thus helping the communication era. Personalized QoS parameters that are based on user's location are an important factor for the recommended techniques of Chen, Zheng, Liu, Huang and Sun [2]. This method is innovative in the way it provides a visual appearance of the available web services, thus easing the user to have a clear idea of the service compositions. But the reality is that the different users in the same location sense a difference in the behavior of the system and as there is no way to include user's feedback to improve the quality of a web service.

When a client submits a request for a web service acquisition, he in turn receives various responses from different Web services with the same functionality. Users compare the services with non-functional property, the Quality of Service (QoS) to choose the best one. The Web Service recommendation methodology Fuzzy C-Means (FCM) [3] uses the fuzzy clustering process to support in QoS prediction. The process forms clusters of web services based on QoS dimensions such as Performance, Strength 
Throughput, Scalability, Reliability and Accuracy and ranks the clusters based on their service indicators Response time, Throughput, Number of operations, Rate of failures and the Rate of errors.

Service performance is unpredictable is most of the scenarios. As discussed above when user preferences are considered for service consumption, it becomes a messy task. This is why because, if the user gives preference for undesired factors of the service quality, it may lead him to undesired results. Hence a novel concept called p-dominant service skyline [4] came to picture to select a service provider S, where the dominant value of service provide $S$ is less than the dominant value $p$.

Quality of Service is used in large to describe the non-functional characteristics of a web service, as discussed by Zhang, Liu, Zhang and Sun [3]. The existing QoS dataset trends are limited to the real-world fact that various users will notice varied QoS values of the same Web service. But there is a lack of real-world QoS datasets for corroborating the emerging QoS based processes and representations of the Web services [5]. Hence re-usable datasets are formed by considering 21,358 real world web services and filtering them based on service failures, HTTP and WSDL errors. Finally a report is generated with a support on Response Time, the time taken to send a request and receive a response, Failure Probability, way to withstand failures and Throughput, total number of invocations for a given period of time.

Service oriented architecture depends more on auto identification and composition of web services. The automated process focuses more on the functionalities of the Web Service. [6].

As discussed above service oriented architecture is more prevalent in current trends and hence enough focus is required on optimization factors of service systems such as robustness, system orientation, and being dynamic and transparent [7].

QoS has been always an important concept of service acquisition. Analyzing QoS values for composite services with complex structures is a really tedious task. Hence the authors Huiyuan Zheng, Weiliang Zhao, Jian Yang, and Athman Bouguettaya [8] focus towards creating a QoS analysis approach.

Selection and composition of web services have remained as an ever challenging task. Hence, as said above QoS comes into the scenario of problem salvation with a focus on execution price, execution duration, reputation, successful execution rate, and availability. This is accompanied with transactional properties of the service composition [9]. An automatic management of virtual servers is achieved by the high - level QOS applications. The existence of potential performance [10] is achieved by the high availability of resource management.

\section{Materials and Methods}

\subsection{QOS Prediction Model}

\subsubsection{Proposed model}

Several Web Service Techniques for QOS modus operandi over the service perhaps the Optimal Incremental Multi Attributes Property Filtering Algorithm to ensure the demand for more efficient access with an Aggregated Quantified Matrix Formulation Algorithm that include caching, perfecting, pushing, and replication over the web service .QoS perceived by its client is thus becoming a prevailing aspect for the Matrix Formulation of the Monitoring QOS-based Web service with the attributes perceiving those related request/response service responsiveness, i.e., the service availability and timeliness. Optimal Property Filtering Algorithm incorporates the Quality of service with its combination of several qualities, including Monitor Response Data, Matrix Formulation, Aggregate responses, Top K Retrieval, Report Generation with the concerned availability, security properties, response time, and throughput. The preponderance of Web services are generally concerned about the levels of quality of service from the perspective of the QOS updating to ARMF Server of these service programs that send requests for both client-side and server-side for achieving QoS improvement in Web server replication, focusing our attention on the client-side 
solutions.

In order to make an accurate QoS prediction, sufficient QoS data from different users are required. However, it is intricate to obtain QoS values from various users. To solve this issue, an Aggregated quantified Response time Matrix (ARMF) is proposed in Fig1. In this approach ARMF uses two algorithms AQMF and OIMF. For quality matrix formulation the AQMF algorithm in turn uses three techniques such as Monitor Response Data, Matrix Formulation and Aggregate Responses. To achieve the QoS data prediction the OIMF consumes two methods called Top-k Retrieval and Report Generation.

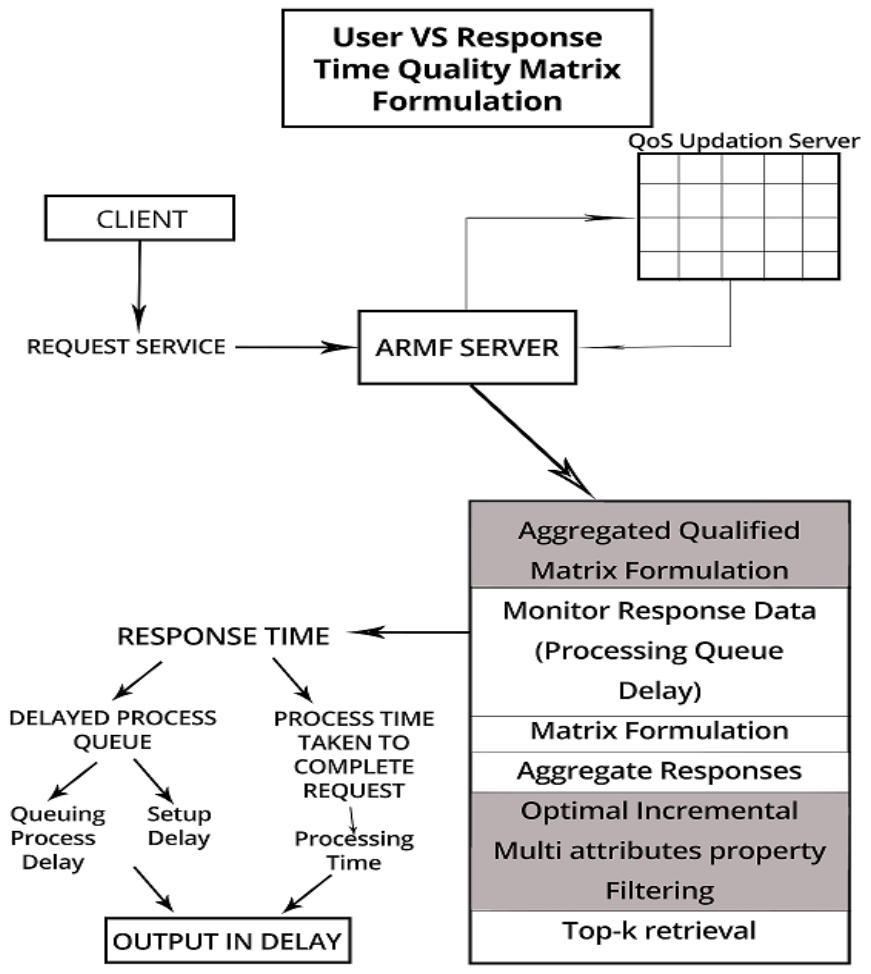

Fig. 1. ARMF based QoS value prediction framework.

\section{Aggregated Quantified Matrix Formulation (AQMF) Technique}

\subsection{Monitoring Response Data}

Monitoring channels might be configured at runtime to change information gathering parameters. Following information are connected with the online checking process

- The recurrence of such information accumulation,

- The decisions made for neighborhood information aggregation (i.e., the exactness of checking information conveyed to remote managers),

- The courses in which information is conveyed to remote managers (i.e., observing granularity), and

- Different QoS metrics.

- Monitoring the data response from each web service and QoS attributes.

Moreover, By Partner QoS qualities with individual observing channels, diverse channels can have distinctive characteristics, subsequently aiding the higher level managers to separate the QoS levels for distinctive checking errands. At last, the final step forms the empowerment of the observing channels to screen the reaction process for the customer preferred web service.

\subsection{Matrix Formulation}


In all actuality, the user-response matrix is generally exceptionally sparse [7], which will extraordinarily impact the prediction accuracy. Predicting missing qualities for the user-response matrix can enhance the prediction accuracy of the dynamic users. As a result, the missing quality prediction approach is proposed for making the matrix denser. By this approach, the user-response matrix gets denser and will be utilized for the missing worth prediction for the dynamic users.

\subsection{Aggregate Response}

We propose an instrument to focus the QoS in a service composition by utilizing a generic workflow to demonstrate the premise of the algorithmic aggregation of nearby QoS measurements. The proposal characterizes the aggregation of QoS, one for each one sample and one for each sort of QoS measurement. Utilizing a hierarchical approach, the aggregated QoS measurements for every composition example, could be aggregated keeping in mind the end goal to give QoS measurements of the entire composition. Finally, there exists an aggregation definition for every fusion of composition examples and QoS measurements.

\section{Optimal Incremental Multi Attributes Property Filtering Technique}

\subsection{Top-k Retrieval}

Top-k is one of the best-performing services prescribed to help the clients to uncover the potential services. Top-k figures out the number of neighbors utilized in the phase of QoS prediction which hence identifies with the prediction accuracy. To study the effect of neighborhood size, top-k value is varied from 5 to 40. The patterns of the three curves are the same that Mean Absolute Error (MAE) decreases pointedly with an expanding neighborhood size at the starting, and afterward stays around a certain quality. At top-k develops, more regions are chosen in the QoS prediction, while those later included regions are normally less comparable and make little commitment to the last come about.

\subsection{Report Generation}

Both the response time and the failure rate, the comparable things are more critical than the comparative users. Constrained Web Services QoS values are given by the active users, while the comparative users become more vital when more QoS values are accessible for the active users. This perception is additionally affirmed by the exploratory results reported. More examinations will be led to QoS worth fixes, since the QoS values of Web services are changing now and again in actuality The web services assessments reported in this approach decreases the impact of the Web services requests to this present reality Web service.

\section{Traditional Approaches and Problem Description}

When a service user prepares to implement a service to develop an enterprise-level application, they have to replace each abstract service with a concrete service. For a service, only a few service implementations will meet the needs of its function. Hence, a sensible way is to select a service with high QoS value. However, for some specific users the QoS values of a particular web service may not be available. As a result, it is imperative to calculate the QoS values of such services based on the existing information. In such cases the proposed approach presents a user-response time matrix to predict the unknown QoS value.

The methodology of Web service QoS value prediction typically computed as follows: when the user request arrives, the process of finding a similar user is first employed. In this process, the collected QoS values are utilized to calculate user similarity using Pearson Correlation Coefficient between the current user and the other users of the same requested service. After similarity calculation, the unknown QoS values are obtained by using a QoS Prediction process. The process incorporates a user-response matrix where every entry in the matrix depicts the QoS value of the service scrutinized by the user. 


\subsection{NIMF vS ARMF}

In neighborhood integrated matrix factorization (NIMF) the process was separated into two parts, the part gained from the characteristic vectors of the client and neighbors, individually. In phase one, similar users are computed using Pearson correlation coefficient and then Top-K recommendations are used to determine the most similar user. In phase two, based on similar user information missing QoS data are obtained using NIMF method. This methodology simply demonstrated the service invocation process from user collaboration. However, the influence of the user's location was ignored. The proposed ARMF methodology incorporates both local and global neighborhood information as well as similar user. This approach provides higher accuracy than the neighborhood integrated matrix factorization.

\section{Aggregated Quantified Response Time Matrix Formulation (ARMF)}

\subsection{Similarity Computation}

Given a $m \times n$ user-response matrix $M$, comprising of $m$ service users and $n$ web services, every entry in this matrix $M_{i j}$ indicates the value of a certain client side QoS property of Web service $\mathrm{j}$ scrutinized by service user i. Assuming that user i did not raise the web service $\mathrm{j}$ in the recent past, then $\mathrm{M}_{\mathrm{ij}}=\emptyset$. Utilizing the accessible Web service QoS values in the user- response time matrix, which are gathered from distinctive service users, the similarities between diverse service users can be calculated by Pearson Correlation Coefficient (PCC).

$$
\begin{gathered}
\operatorname{PCC}(i, j)=\frac{\sum_{k \in K}\left(M_{i k}-M_{i}^{\prime}\right)\left(M_{k j}-M_{k}^{\prime}\right)}{\sqrt{\sum_{k \in j}\left(M_{i k}-M_{i}^{\prime}\right)^{2} \sqrt{\sum_{k \in K}\left(M_{j k}-M_{j}^{\prime}\right)^{2}}}} \\
\operatorname{PCC}(i, j)=\frac{\sum_{k \in K}\left(\left|M_{i k}\right|\right) \mid\left(M_{j k} \mid\right)}{\sqrt{\sum_{k \in j}|M i k|^{2} \sqrt{\sum_{k \in k}|M j k|^{2}}}}
\end{gathered}
$$

where $k$ is the subset of Web services which are raised by both user $i$ and user $j . M_{i j}$ is the QoS quality of Web service $k$ observed by service user $i$, and $M_{i}$ and $M_{k}$ is the normal QoS values of distinctive Web services scrutinized by service user $i$ and $j$, individually.

After estimating the similarities, a set of Top-K similar user are obtained utilizing the PCC values. In practice, a service user may have predetermined number of comparative users. Traditional Top-K algorithms disregard this issue and still incorporate unique clients with negative PCC values, which will significantly impact the prediction precision. Thus, the different service users who have negative PCC values are eliminated.

\subsubsection{Pseudo code}

Step 1: Service Consumer raises the Service Request from the access point

Step 2: Service Selection process for the Invoked Service Request

Step 3: Provide service from the available and suitable web service

Step 4: Identification Of Similarity of the service provision.

Step 5: Matrix Monitorer acquires the Utility Web Method allocation of Service Provider Pearson Correlation Coefficient (PCC). 
Step 6: Subset $k$ is raised and monitored for all users $i$ and $j$.

Step 7: Identification Of Top-K similar user from (PCC).

Step 8: Identification Of unique user $i$ and $j$ with negative PCC

Step 9: Eliminate the service with negative PCC for user $i, j$

Step 10: Store the Resultant as Pearson Correlation Coefficient (PCC).

Step 11: Compare the available matrix with Reliability And Availability Measure check with the generated responses.

\subsection{QoS Prediction}

A prominent methodology to anticipate missing values is to fit a component model to the user-response time matrix, and utilize this element model to make further predictions. In our methodology, we eliminate the different service users who have negative PCC values. For a user, the set of similar users have web services which are obtained by both users. And we eliminate the different services and can design our aggregated quantified. The reason behind a low-dimensional element model is that there is a little number of components impacting the QoS utilization information, and that a user's QoS utilization information on a Web service is controlled by how each variable applies to the user and the Web service.

\section{Results and Discussion}

\subsection{Metrics}

To assess the prediction accuracy's for our technique, we compute the average values of Mean Absolute Error (MAE) and Root Mean Square Error (RMSE) dependent upon the predicting effects. The RMSE is defined as follows. Here, the square of the difference between the predicted QoS value and the corresponding observed value are performed and the result is averaged. At the end, the square root of the average value is done. RMSE is most suitable when large errors are especially adverse.

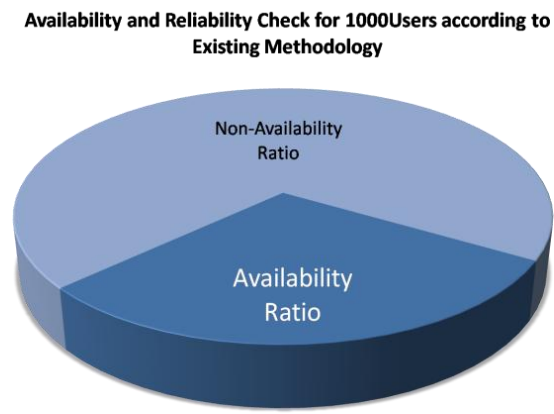

\begin{tabular}{|c|c|}
\hline Non-Availability Ratio & Availability Ratio \\
\hline 1162 & 2732 \\
\hline
\end{tabular}

Fig. 2. Availability and reliability Check for 1000 users according to existing methodology.

Fig. 2 and Fig. 3 depicts the availability and Reliability of Web services for 1000 user's requests of Existing and Proposed methodologies by comparing the availability and unavailability ratio of the web services per 1000 users. Fig. 4 and Fig. 5 depict the quality of service of considered web services such as Authentication related web services and MAC Address fetches Webservices according to the existing and proposed Methodologies. The ratio of QOS of web service, according to the existing methodology is $(20,95,90,0)$ per 1000 users and the ratio of QOS of web service, according to the proposed methodology is $(125,30,270,130)$ per 1000 users. Hence the resulting ratio of the comparison chart of the proposed methodology is effective than existing as the reliability of the web service count is much better than the existing. 


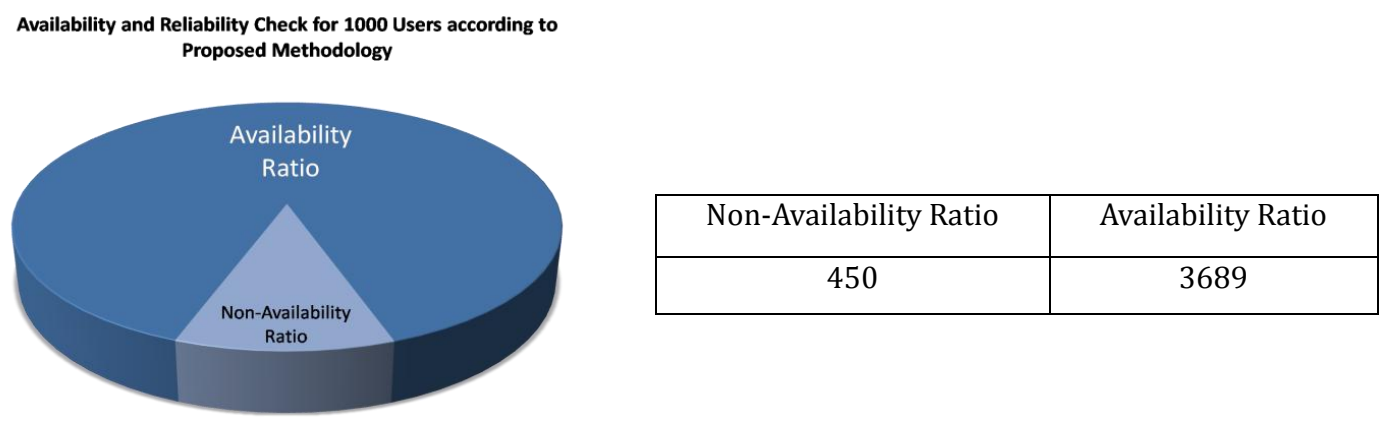

Fig. 3. Availability and reliability check for 1000 Users according to proposed methodology.

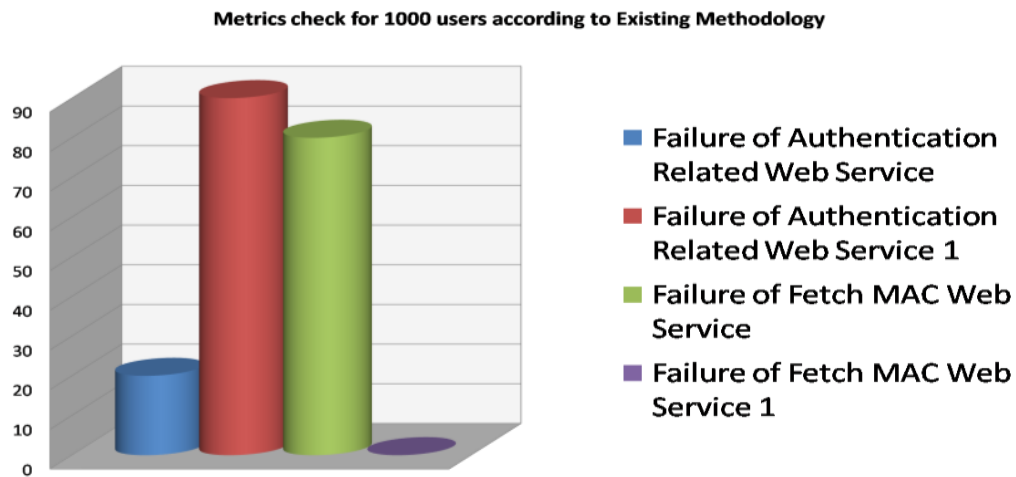

Fig. 4. Reliability metrics check for 1000 users according to existing methodology.

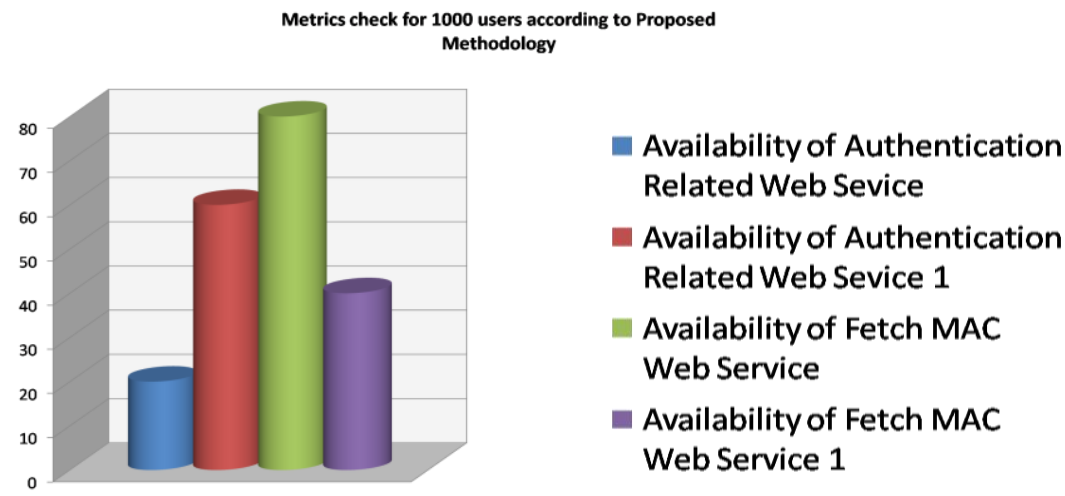

Fig. 5. Reliability metrics check for 1000 users according to proposed methodology.

\section{Conclusion}

Based on the instinct that a user's Web service QoS utilization information could be anticipated by both the user's qualities and the past usage information of other similar users, an Aggregated Quantified Response time Matrix formulation approach is proposed for making the customized QoS value prediction. It provides the user-response matrix where every entry in the matrix depicts the QoS value of the service opted by the user.

ARMF approach as well utilizes the collaborative methodologies to attain higher prediction accuracy. The experimental results show the effectiveness of our methodology. In the event of acquiring the anticipated QoS values on the unused Web services, most service users will make invocations to the chosen Web services. The QoS values of these Web service invocations hold important data for enhancing the QoS prediction accuracy. 


\section{References}

[1] Dario, B., Salvatore, D., F. L., \& Marco, S. (2013). Stochastic evaluation of QoS in service-based systems. IEEE Transactions on Parallel and Distributed Systems, 24(10).

[2] Xi, C. et al. (2013). Personalized QoS-aware web service recommendation and visualization. IEEE Transactions on Services Computing, 6(1).

[3] Meng, Z. et al. (2012). A web service recommendation approach based on QoS prediction using fuzzy clustering. Proceedings of the 2012 IEEE Ninth International Conference on Services Computing.

[4] Qi, Y., \& Bouguettaya, A. (2010). Computing service skyline from uncertain QoWS'. IEEE Transactions on Services Computing, 3(1).

[5] Zheng, Z., Zhang, Y., \& Lyu, M. R. (2010). Distributed QoS evaluation for real-world web services. Proceedings of the 2010 IEEE International Conference on Web Services.

[6] Kyriakos, K., \& Dimitris, P. (2009). Requirements for QoS-based web service description and discovery. IEEE Transactions on Services Computing, 2(4).

[7] Liang, Q. H., Wu, X. D., \& Lau, H. C. (2009). Optimizing service systems based on application-level QoS. IEEE Transactions on Services Computing, 2(2).

[8] Zheng, H. Y., Zhao, W. l., Yang, J., \& Athman, B. (2013). QoS analysis for web service compositions with complex structures. IEEE Transactions on Services Computing, 6(3).

[9] Joyce, E. H., Maude, M., \& Marta, R. TQoS: Transactional and QoS-aware selection algorithm for automatic web service composition. IEEE Transactions ON Services Computing, 3(1).

[10] Gokul, R. T., \& Anbumani, A. (2014). Autonomic virtual resource and workload management applying nefeli. International Journal of Inventions in Computer Science and Engineering, 1(3).

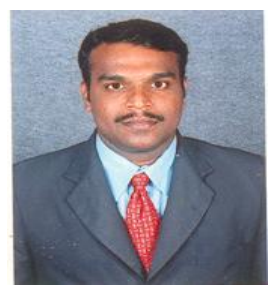

Balika. J. Chelliah was born in Tamil Nadu, India in 1982. He received the master degree of computer science engineering from SRM University, Chennai, India in 2006, and is pursuing the Ph.D. degree of computer science engineering from SRM University, Chennai, India. Now he is a asst. professor of SRM University, and his major fields of study are web services, service oriented architecture.

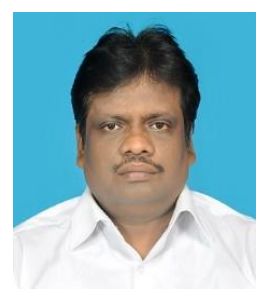

K. Vivekanandan obtained his B.E degree in electronics and communication engineering from Coimbatore Institute of Technology in 1986, the M.Tech in computer science and engineering from Indian Institute of Technology in 1991 and the Ph.D in 2005 from Pondicherry University in the area of software engineering. His teaching, research and development interests have been in the areas of software engineering, object oriented systems, DBMS and data warehousing, Information security and data mining. 